\title{
THREE-DIMENSIONAL KINEMATIC ANALYSIS OF STEPPING OVER OBSTACLES IN YOUNG SUBJECTS
}

\author{
HAO-LING Chen, Tung-Wu Lu and H.C. LIN
}

Institute of Biomedical Engineering, National Taiwan University, Taiwan

ABSTRACT
A better understanding of the kinematics and control strategies adopted during obstacle
crossing is essential for the prevention of injuries associated with falls in the elderly. The effects of
obstacle height on the foot clearance, foot-obstacle distance and joint kinematics have presented
significant controversy. This may be related to the selection of obstacle height, whether the obstacles
are normalized to the leg length, and to the calculation of the foot-obstacle clearance, as well as the
extraction of representative joint angles for the analysis. In this study, fourteen young healthy adults
wearing 28 infrared retroreflective markers walked and crossed obstacles of heights of $0 \%, 10 \%$,
$20 \%$ and $30 \%$ of their leg lengths in a gait laboratory equipped with a $3 D$ motion analysis system.
Three-dimensional joint kinematics of the lower limb were calculated. Foot clearances were
calculated using the heel and toe markers. The results suggested that young subjects maintained a
constant margin of leading foot clearance when crossing higher obstacles (higher than $79.4 m m$ and
a constant trailing foot clearance for all obstacle heights. Both toe-obstacle and heel-obstacle
horizontal distances were not affected by obstacle height. Apart from the peak values, kinematic
variables for the leading limb should be considered both when the toe and heel cross the obstacle
while only those when the toe crosses the obstacle for the trailing limb. Not only in the sagittal
plane, motions of the lower limb in the other two planes were also important when investigating the
kinematics of the leading limb during obstacle crossing. The present study clarified some of the
controversies in the literature of obstacle-crossing through careful comparisons of kinematic
variables obtained from different study aspects. The results will be helpful for future studies to gain
insight into the kinematics and control strategies adopted during obstacle-crossing.
Biomed Eng Appl Basis Comm, 2004(June); 16 : $157-164$.
Keywords: Gait, obstacles, foot clearance, joint angles, lower limb

\section{INTRODUCTION}

There have been research interests on the analysis of the kinematics of the human locomotor system during crossing obstacles [1-6], due to its significant relevance with the falls in the elderly. About $25 \%$ to $35 \%$ of people over 65 -year-old fall each year [7-9], a

Received: Jan 7, 2004; Accepted: Mar 25, 2004

Correspondence: Tung-Wu Lu, Professor

Institute of Biomedical Engineering, National Taiwan

University, Taipei, Taiwan

E-mail: twlu@ntu.edu.tw large proportion of whom tripped over obstacles during gait. A better understanding of the kinematics of obstacle crossing and the control strategies adopted during this functional activity is essential for the prevention of associated injuries.

Foot (or foot-obstacle) clearance has been used as an important kinematic index for the evaluation of the strategies used during crossing obstacles of different height. The effects of obstacle height on foot clearance have been a subject of extensive research and have presented significant controversy. For the leading limb, Chen et al. [2] used the lowest of the heel, toe and mid-foot markers to calculate the foot clearance when stepping over obstacles of 3 different heights $(25,51,152 \mathrm{~mm})$. They found that in $82 \%$ of the trials, 
the leading heel was the lowest and that foot clearance increased significantly with increasing obstacle height. Sparrow et al. [6] suggested that potential errors in clearance calculations could arise from subject size differences so obstacles should be adjusted for each subject according to their leg lengths. They used the heel marker to study the effects of obstacle height $(10$, $25,40 \%$ leg length) on foot clearance and found that foot clearance was not affected by obstacle height. Patla et al. [5] used the toe marker to calculate the foot clearance when crossing obstacles with 3 heights $(67$, $134,268 \mathrm{~mm}$ ). No significant difference was found between the clearances in 67 and 134 obstacles but that for the $268 \mathrm{~mm}$ obstacle was higher than those for the lower obstacles. Austin et al. [1] used both toe and heel markers to calculate the foot clearance when crossing obstacles of three heights $(31,76,126 \mathrm{~mm})$ and found that both toe and heel clearances increased with obstacle height except for 76 and $126 \mathrm{~mm}$ obstacles. For the trailing limb, Sparrow et al. [6] used the heel marker to study the effects of obstacle height $(10,25,40 \%$ leg length) on foot clearance and found that the heel clearance increased with obstacle height. Chou et al. [3] used the toe marker to calculate the trailing foot clearance when crossing obstacles with 4 different heights $(51,102,153,204 \mathrm{~mm})$ and found that the foot clearance was not affected by obstacle height. From the literature review, it revealed that the effects of obstacle height on foot clearance, both leading and trailing, during obstacle-crossing remain controversial. It may be due to the difference in the selection of obstacle height, either discrete fixed heights or adjusted to subject leg length, and/or the markers used for the calculation of the foot clearance.

Strategies during obstacle-crossing can be further studied by examining the angular kinematics of the lower extremities. In the majority of previous studies only sagittal plane components of the joint angles were presented (e.g. McFadyen et al. [4] ). Some used peak values of these joint angles for statistical analyses. For example, Austin et al. [1] investigated the effects of obstacle height on joint kinematics using maximum sagittal joint angles of the leading foot. Although joint angles when the foot was above the obstacle may be important in studying the obstacle height effects, few studies adopted these variables for their studies. Patla et al. [5] reported joint angles of the leading leg in the sagittal plane when the toe was over the obstacle. The flexion angles of the hip and knee were found to increase with obstacle height $(67,134$, $268 \mathrm{~mm}$ ), while the ankle dorsiflexion angles were not affected by the two lower obstacles but were significantly higher in the highest condition.

Joint angles in the other two planes may reveal important information. Chou et al. [3] investigated 3dimensional joint angles of the trailing limb when the toe was over the obstacles $(51,102,153,204 \mathrm{~mm})$. The flexion angles of the hip and knee were found to be affected by obstacle height but the other two components were not. In a recent study, McFadyen et al. [10] found that all three joint motion components of both the leading and trailing legs were affected by obstacle type and subject age. However, the study was limited to only one obstacle height $(117.5 \mathrm{~mm})$ so the effects of obstacle height on the 3 rotational components of the leading limb joints could not be studied.

Controversies exist in the literature regarding the effects of obstacle height on the lower limb kinematics. This can be related to the selection of obstacle height, whether the obstacles are normalized to the leg length, and the calculation of the foot-obstacle clearance, as well as the extraction of representative joint angles for subsequent analysis. Therefore, the main purpose of the present study was to investigate the strategies adopted when crossing obstacles with different heights in normal young adults in terms of the kinematics of the leading and trailing limbs, including foot clearance, 3D joint angles and foot-obstacle horizontal distances. Obstacles with different heights that were normalised to the subject's leg length were used in the study to eliminate effects from normal anatomical variations. Three methods for the calculation of foot clearance were compared. Selection of key features of joint angle curves for statistical analysis was also studied.

\section{MATERIALS AND METHODS}

Fourteen young adults (age: $23.2 \pm 4.9$ years, height: $171.7 \pm 5.8 \mathrm{~cm}$, weight: $70.8 \pm 7.1 \mathrm{~kg}$ ) participated in the present study with informed consents. They were all free of neuromusculoskeletal dysfunction and with normal corrected vision. In a gait laboratory, twenty-eight infrared retroreflective markers were used to track the motion of the limb segments, namely the pelvis (ASIS's, PSIS's) and each thigh (greater trochanter, mid-thigh, medial and later epicondyles), shank (head of fibula, tibial tuberocity, medial and lateral malleolus), and foot (navicular tuberosity, $5^{\text {th }}$ metatarsal base, big toe and heel). Each subject walked at self-selected pace and crossed a height-adjustable obstacle made of an aluminium tube placed across a metal frame, Fig. 1. The subjects were allowed to familiarize themselves with the walkway before experimental data were taken. Kinematic data were measured with a 6-camera motion analysis system (Vicon512, Oxford Metrics, U.K.). Two forceplates (AMTI, Advanced Mechanical Technology, U.S.A.) were placed on each side of the obstacle so that the ground reaction forces (GRF) of each foot could be measured. Test conditions included 
unobstructed gait and crossing obstacles of three different heights (10,20 and $30 \%$ of leg length) for both limbs. For all conditions, subjects were instructed to walk along the walkway and step over the obstacle when necessary. Six trials, three for each leg, for each condition were recorded.

The measured forceplate data were used to define the cycle of obstacle-crossing and marker data to calculate kinematic variables for each test condition, including foot-obstacle distances, foot clearances and joint angles. The trailing foot-obstacle distance was defined as the horizontal distance between the toe marker and the obstacle, also called toe-obstacle distance, Fig.1. The leading foot-obstacle distance was defined as the horizontal distance between the heel marker and obstacle, also called heel-obstacle distance, Fig.1. Three possible definitions of foot-clearance were used, namely heel clearance calculated using the heel marker, toe clearance using toe marker and minimum clearance calculated as the smaller of the former two values. Each body segment was embedded with an orthogonal coordinate system with the positive $\mathrm{x}$-axis directed anteriorly, the positive $y$-axis superiorly and the positive $\mathrm{z}$-axis to the right. Cardanic rotation

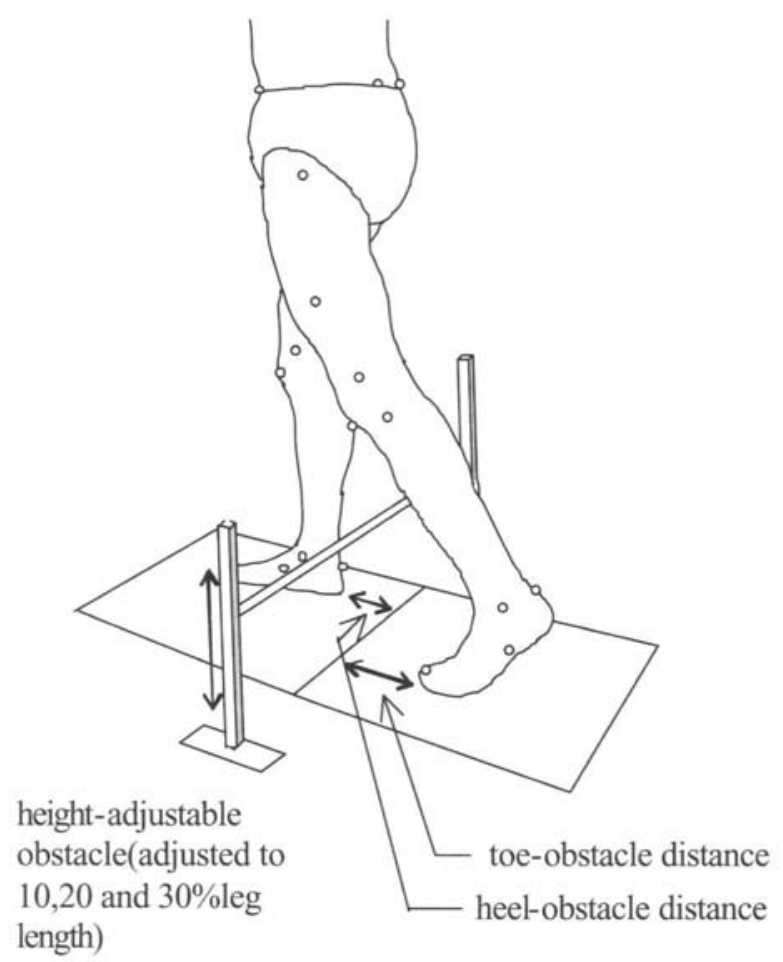

Fig. 1. Schematic diagram showing a subject crossing a height-adjustable obstacle with each foot on one forceplate, while the 3D trajectories of the markers on the limb segments are measured by a 6camera motion analysis system (not shown). sequence $(\mathrm{Z}-\mathrm{Y}-\mathrm{X})$ was used to describe the rotational movements of each joint (Grood and Suntay, [11]). The peak angles during the gait cycle as well as angles when the heel and toe markers were above the obstacle for each joint were calculated for further statistical analysis. The calculated variables were compared using repeated measures t-test. With Bonferroni's correction for multiple comparisons the significance level was set at 0.016 .

\section{RESULTS}

None of the toe, heel and minimum clearance distances for both the leading and trailing legs were affected by obstacle height but all were significantly higher than the minimum clearance during level waking $(\mathrm{p}<0.016$, Table 1$)$. The leading toe and heel clearance distances were not significantly different, indicating that the leading foot was moving almost horizontally without rotation when crossing the obstacle. Toe clearance distances of the leading and trailing limbs were indifferent for all heights $(p<0.05)$, indicating that there were equal chances of tripping over the obstacle for both limbs. The toe-obstacle horizontal distances of the trailing leg were $180.73 \pm$ $31.48,160.31 \pm 39.50$ and $166.88 \pm 28.45 \mathrm{~mm}$ for 10 , 20 and $30 \%$ obstacles, respectively. Corresponding values for the heel-obstacle horizontal distances of the leading leg were $196.36 \pm 31.48,198.16 \pm 23.89$ and $196.28 \pm 20.71 \mathrm{~mm}$.

The flexion/extension, adduction/abduction and internal/external rotation displacements of the joints of the leading limb when crossing obstacles are shown in Fig. 2. Corresponding data for the trailing leg are given in Fig. 3. It is noted that the main movements of the joints occurred in the sagittal plane. The maximum angular displacements in the sagittal plane of the knee and ankle joints occurred before the leading toe crossed the obstacle $(p<0.016)$ while that of the hip occurred between the leading toe and heel crossing over the obstacle $(p<0.016)$, Fig. 2 . For the trailing limb, the maximum sagittal angular displacements all occurred after the trailing foot (both toe and heel markers) passed over the obstacle $(p<0.016)$, Fig. 3 . The time needed for the toe and heel to cross the obstacle in the leading limb was significantly longer than that of the trailing $\operatorname{limb}(\mathrm{p}<0.016)$.

Maximum angular displacements of the hip, knee and ankle joints when crossing obstacles for the leading and trailing limbs are shown in Figs. 4 and 5, respectively. The maximum angular displacement in the sagittal plane of the leading hip and knee joint increased significantly with increasing obstacle height $(\mathrm{p}<0.016)$ while there was no significant difference in ankle joint angles for all height comparisons, Fig.4. In 
Table 1. Toe, heel and minimum clearance distances for both the leading and trailing legs when crossing obstacles of three heights

\begin{tabular}{cllll}
\hline & \multicolumn{4}{c}{ Obstacle Height } \\
Clearance $(\mathrm{mm})$ & $0 \% \mathrm{LL}$ & $10 \% \mathrm{LL}$ & $20 \% \mathrm{LL}$ & $30 \% \mathrm{LL}$ \\
\hline Lead Heel & - & $117.18(35.38)$ & $128.64(27.43)$ & $128.77(26.38)$ \\
Toe & - & $121.79(27.42)$ & $124.68(14.75)$ & $121.46(12.64)$ \\
Min & $55.38(7.78)$ & $108.23(30.72)$ & $118.36(15.87)$ & $115.82(12.27)$ \\
\hline Heel & - & $330.00(38.90)$ & $336.73(42.63)$ & $332.85(50.34)$ \\
Trail Toe & - & $113.28(36.30)$ & $118.31(41.95)$ & $124.48(50.52)$ \\
Min & $53.94(10.44)$ & $113.28(36.30)$ & $118.31(41.95)$ & $124.48(50.52)$
\end{tabular}

the frontal plane, the maximum leading knee adduction increased significantly with increasing obstacle height while the hip abduction in the $30 \%$ condition was significantly bigger than those in the $20 \%$ and $10 \%$ conditions $(\mathrm{p}<0.016)$, Fig.4 (a) (b). The maximum leading ankle abduction angles were not different in all heights $(p>0.016)$. In the transverse plane, the maximum internal rotation of the ankle in the $30 \%$ condition was significantly bigger than that in the $10 \%$ condition $(p<0.016)$, Fig. 4 (c) while there was no difference for the other two joints $(p>0.016)$. The maximum knee flexion of the trailing limb increased significantly with obstacle height $(\mathrm{p}<0.016)$, Fig.5 (b). The maximum hip flexion angle in the $30 \%$ condition was significantly bigger than that in the $10 \%$ and $20 \%$ conditions while the maximum ankle dorsiflexion in the $30 \%$ condition was significantly bigger than that in the $10 \%$ condition $(p<0.016)$, Fig. 5 (a)(c). The maximum angular displacements in the frontal plane of all joints were not affected by obstacle height. The maximum knee external rotation in the $10 \%$ condition was significantly smaller than those in the $20 \%$ and $30 \%$ conditions, Fig. 5 (b). However, there were no differences among all height pairs for the trailing hip and ankle joints ( $p>0.016$ ), Fig.5 (a) (c).

Angular displacements of the hip, knee and ankle joints when the toe and heel were above the obstacle for the leading and trailing limbs are shown in Figs. 6 and 7 , respectively. For the leading limb, the hip and knee flexion angles increased significantly with increasing obstacle height when the toe and heel were above the obstacle $(p<0.016)$, but the ankle dorsiflexion was not affected by the obstacle height ( $p>0.016$ ), Fig. 6 . The hip abduction angle increased significantly with increasing obstacle height when the toe and heel of the leading limb were above the obstacle $(p<0.016)$, Fig. 6 (a). When the toe was above the obstacle the knee adduction angle in the $30 \%$ condition was significantly bigger than those in the $20 \%$ and $10 \%$ conditions $(p<0.016)$. The knee adduction angle when the heel was above the obstacle increased significantly with obstacle height increased $(\mathrm{p}<0.016)$, Fig. 6 (b). The ankle abduction angle was not affected by obstacle height at the time when the toe was above the obstacle. But, the ankle abduction in the $30 \%$ condition was significantly bigger than that in the $10 \%$ condition at the time when the heel was crossing $(\mathrm{p}<0.016)$, Fig. $6(\mathrm{c})$. When the toe was above the obstacle, the ankle internal rotation in the $30 \%$ condition was significantly bigger than that in the $10 \%$ condition $(p<0.016)$ while there were no differences for the hip and knee for all heights ( $p>0.016$ ), Fig. 6 . When the heel was above the obstacle, the hip external rotation angle in the $30 \%$ condition was significantly smaller than that in the $20 \%$ condition $(p<0.016)$, Fig. 6 (a).

For the trailing limb, the heel clearances were significantly much higher than the toe clearances for all heights $(p<0.016)$. The instances at which the heel and toe markers were above the obstacle were not significantly different ( $p>0.016)$ so the kinematics of the joints were indifferent both when toe and heel crossed the obstacle. Therefore, joint variables when the toe was on the obstacle were used for the description of the kinematics of the trailing limb, Fig. 7. When the foot was above the obstacle, the trailing hip flexion angle in the $30 \%$ condition was significantly bigger than that in the $10 \%$ condition $(p<0.016)$ while the knee flexion angle increased significantly with obstacle height increased $(p<0.016)$, Fig. 7 (a) (b). The ankle dorsiflexion angle in the $30 \%$ condition was significantly bigger than those in the $20 \%$ and $10 \%$ conditions $(p<0.016)$, Fig. 7 (c). None of the frontal plane components of the joint angles were affected by obstacle height $(p>0.016)$. Similar results were found for transverse plane components, 

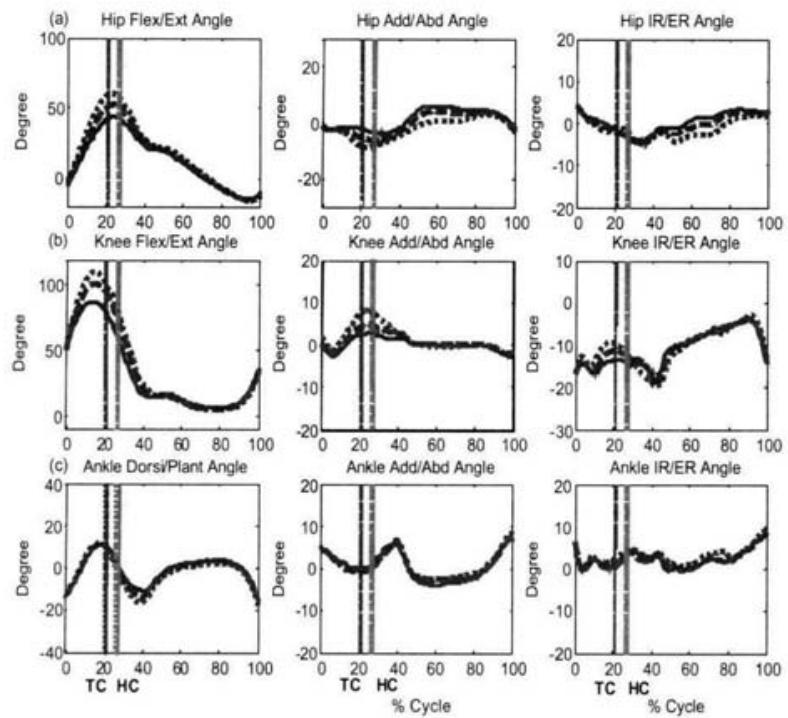

Fig. 2. Three-dimensional angular displacements of the (a) hip, (b) knee and (c) ankle joints of the leading limb when crossing obstacles of $10 \%$ (solid lines), $20 \%$ ( dashed lines) and $30 \%$ (dotted lines) of leg length.( TC: toe above obstacle; HC: heel above obstacle)
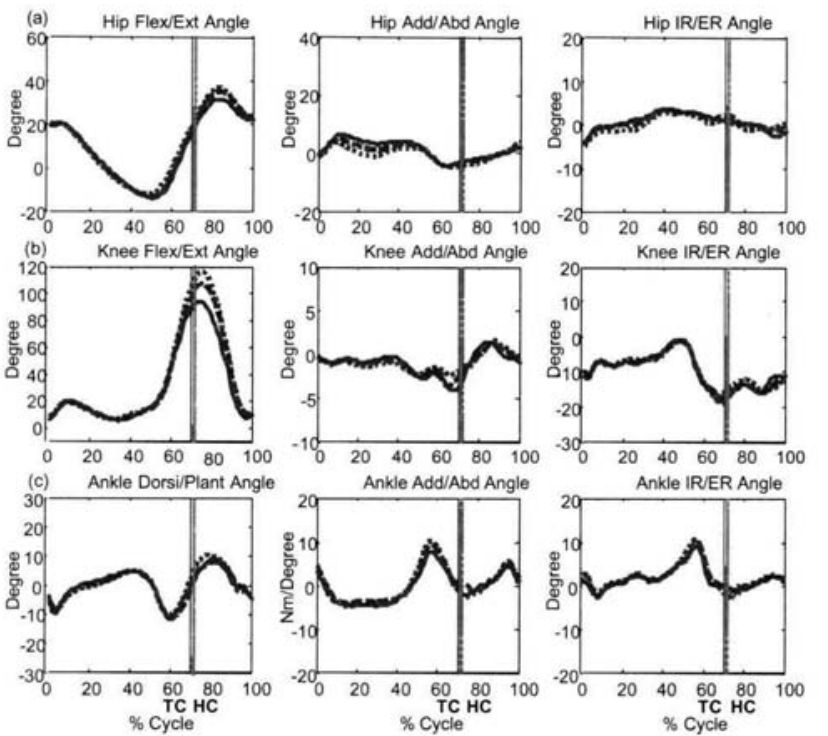

Fig. 3. Three-dimensional angular displacements of the (a) hip, (b) knee and (c) ankle joints of the trailing limb when crossing obstacles of $10 \%$ (solid lines), $20 \%$ (dashed lines) and $30 \%$ (dotted lines) of leg length. ( TC: toe above obstacle; HC: heel above obstacle)

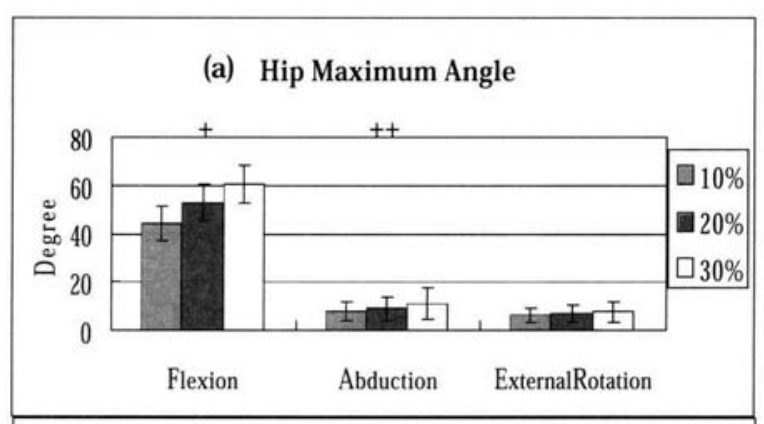

(b) Knee Maximum Angle

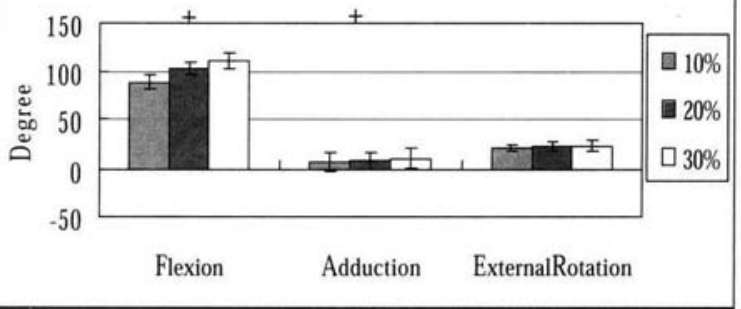

(c) Ankle Maximum Angle

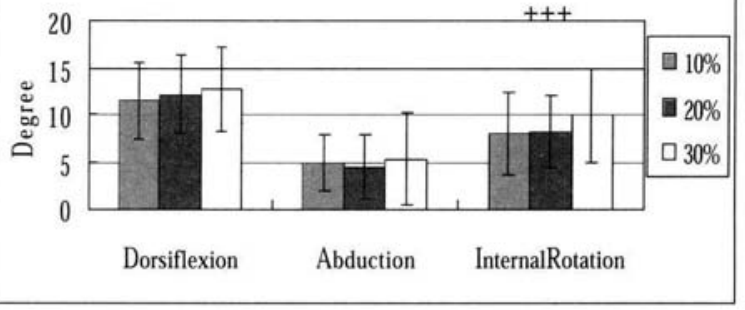

Fig. 4. Maximum angular displacements of the (a) hip, (b) knee and (c) ankle joints of the leading limb when crossing obstacles. (+: significant difference between all two height comparisons; ++: $30 \%>20 \%, 30 \%>10 \%$; ++ : $30 \%>10 \% ; p<0.016$ )

except for the knee joint. The knee external rotation in the $20 \%$ condition was significantly bigger than that in the $10 \%$ condition ( $p<0.016)$, Fig. 7 (b).

\section{DISCUSSION}

Heel, toe and minimum clearances were calculated for three different obstacle height conditions $(10,20$ and $30 \%$ leg length, corresponding to 79.4 , 158.8 and $238.2 \mathrm{~mm}$ for an averaged subject in the study). The results showed that all the clearances, heel, toe or minimum clearances, were not affected by obstacle height $(p>0.016)$, which were in agreement with Sparrow et al.[6] for the leading limb and Chou et 


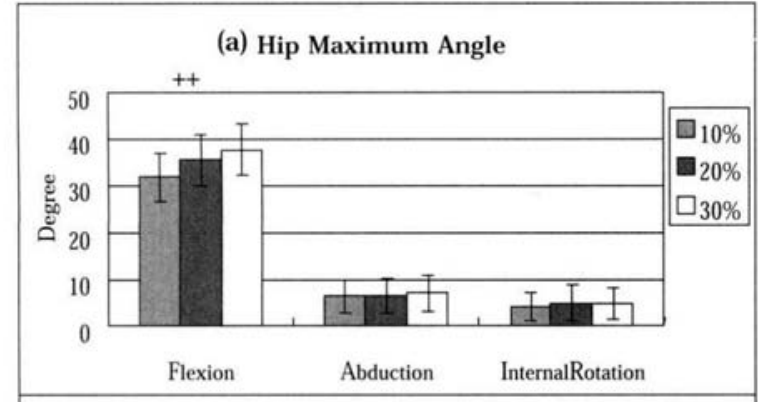

(b) Knee Maximum Angle

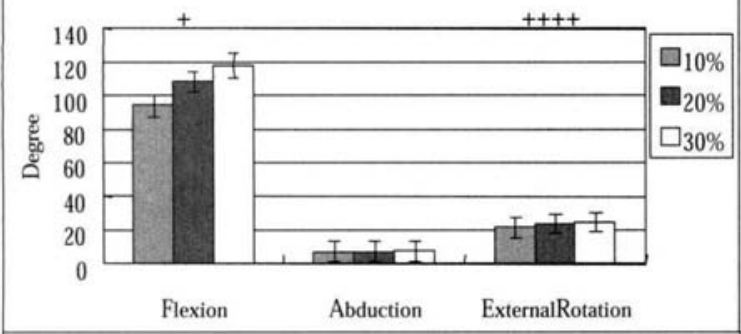

(c) Ankle Maximum Angle

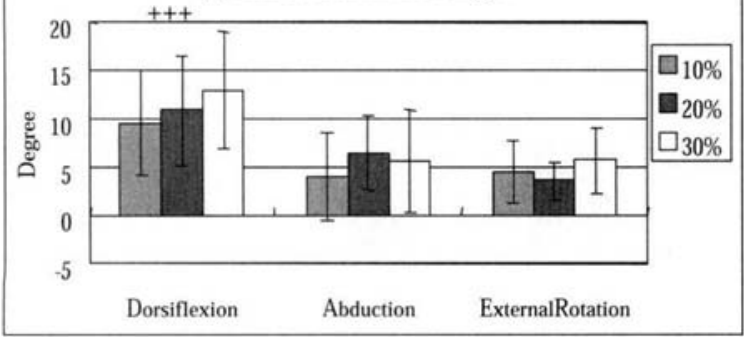

Fig. 5. Maximum angular displacements of the (a) hip, (b) knee and (c) ankle joints of the trailing limb when crossing obstacles. (+: significant difference between all two height comparisons; ++ : $30 \%>20 \%, 30 \%>10 \%$; +++: $\mathbf{3 0} \%>10 \%$; ++++: $30 \%>20 \%, 20 \%>10 \% ; p<0.016$ )

al. [3] for the trailing limb. These results suggested that young subjects maintained a constant margin of leading foot clearance when crossing higher obstacles (higher than $79.4 \mathrm{~mm}$ ). Similar results were also found in Austin et al. [1]. For lower obstacles that were not included in the present study, leading foot clearances may change for different obstacles [1-2]. Further study on obstacle heights smaller than $10 \%$ leg length may be necessary to clarify this point. For toe-obstacle and heel-obstacle horizontal distances, no influence was found from obstacle height, in agreement with the literature (e.g. Austin et al., [1] ).

A successful crossing required sufficient foot clearances for both the toe and heel, which were determined by the angular motion of the lower limb joints. For different obstacles, we found that the hip

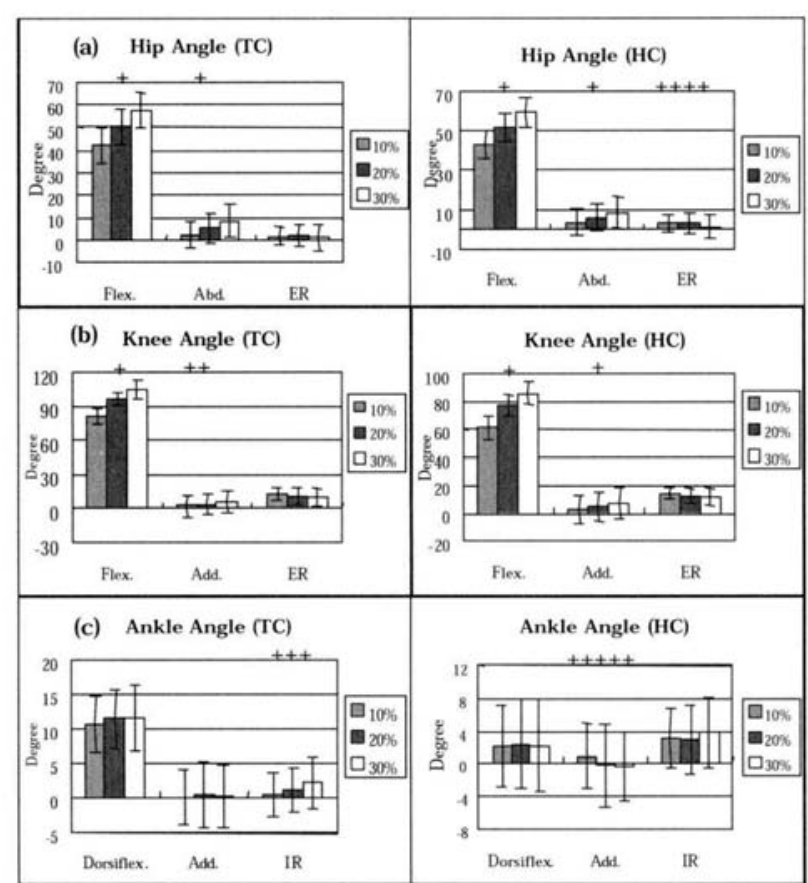

Fig. 6. Angular displacements of the (a) hip, (b) knee and (c) ankle joints of the leading limb when the toe and heel were above the obstacle. (+: significant difference between all two height comparisons; ++: $\mathbf{3 0} \%>\mathbf{2 0} \%, \mathbf{3 0} \%>\mathbf{1 0} \%$; +++ : $30 \%>10 \%$; ++++: $30 \%<20 \%$; +++++: $30 \%<10 \%$; p<0.016; TC: toe above obstacle; HC: heel above obstacle)

and knee were the joints that were adapted to maintain the constant margin of foot clearance, through motions mainly in the sagittal and frontal planes. The leading knee flexion reached its maximum before toe-crossing while the maximum hip flexion occurred after toe- but before heel-crossing. With obstacle height increased, the hip and knee joint flexed more in order to maintain constant clearances when both toe and heel were above the obstacles, in agreement with Patla et al. [5]. The maximum flexions of the hip and knee, which occurred at different times from those for toe- or heel-crossing, also increased with increasing obstacle height, in agreement with Austin et al. [1]. The motion of the hip and knee in the frontal plane also contributed to the maintenance of the constant leading foot clearance when stepping over obstacles of different height. The higher the obstacle was, the bigger the hip abduction and knee adduction. During leading limb crossing, there was a trend that reduced hip external rotation and increased ankle internal rotation were adopted for sufficient foot clearance. Therefore, not only in the sagittal plane, motions of the lower limb in the other 


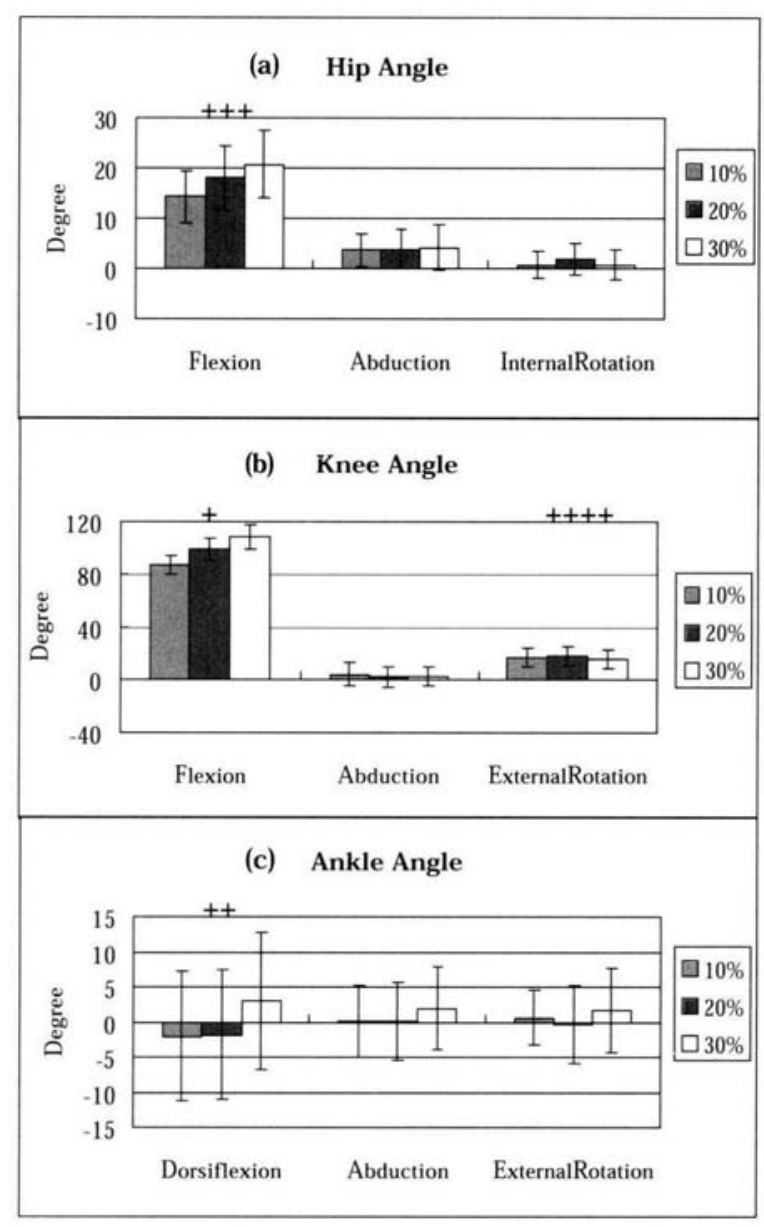

Fig. 7. Angular displacements of the (a) hip, (b) knee and (c) ankle joints of the trailing limb when the foot was above the obstacle. (+: significant difference between all two height comparisons; ++ : $30 \%>20 \%, 30 \%>10 \% ;+++: 30 \%>10 \% ;++++$ : $20 \%>10 \% ; p<0.016$ )

two planes are also important when we investigate the kinematics of the leading limb during obstacle crossing.

During the trailing limb crossing, the maximum flexions of all the three joints occurred after toe and heel had crossed the obstacle. Only the knee flexion increased with obstacle height increased. This suggested that the knee was the primary joint used to adapt to the change of obstacle height. For lower obstacles, moderate ankle plantarflexion of the trailing limb was sufficient to achieve necessary toe clearance for safe crossing in young subjects. However, for higher obstacles, ankle dorsiflexion and bigger hip flexion would be necessary.

\section{CONCLUSION}

The results of the present study suggested that young subjects maintained a constant margin of leading foot clearance when crossing higher obstacles (higher than $79.4 \mathrm{~mm}$ ) and a constant trailing foot clearance for all obstacle heights. Both toe-obstacle and heel-obstacle horizontal distances were not affected by obstacle height. Apart from the peak values, kinematic variables for the leading limb should be considered both when the toe and heel cross the obstacle while only those when the toe crosses the obstacle for the trailing limb. Not only in the sagittal plane, motions of the lower limb in the other two planes were also important when investigating the kinematics of the leading limb during obstacle crossing. The present study clarified some of the controversies in the literature through careful comparisons of kinematic variables obtained from different study aspects. The results of the present study will be helpful for future studies on to gain insight into the kinematics and control strategies adopted during obstacle-crossing.

\section{REFERENCES}

1. G. P. Austin, G. E. Garrett and R. W. Bohannon: Kinematic analysis of obstacle clearance during locomotion. Gait and Posture, 1999; 10: 109-120.

2. H. Chen, J. Ashton-Miller, N. Alexander and A. Schultz: Stepping over obstacles: gait patterns of healthy young old adults. Journal of Gerontology, 1991; 46: M196-203.

3. L.-S. Chou and L. F. Draganich: Stepping over an obstacle increases the motions and moments of the joints of the trainling limb in young adults. Journal of Biomechnics, 1997; 30: 331-337.

4. B. McFadyen and H. Carnahan: Anticipatory locomotor adjustments for accommodating versus avoiding level changes in humans. Experimental Brain Research, 1997; 114: 500-506.

5. A. Patla and S. Rietdyk: visual control of limb trajectory over obstacles during locomotion: effect of obstacle height and wodth. Gait and Posture, 1993; 1: 45-60.

6. W. Sparrow, A. J. Shinkfield, S. Chow and R. Begg: Characteristics of gait in stepping over obstacles. Human Movement Science, 1996; 15: 605-622.

7. R. W. Sattin: Falls among older persons: a public health perspective. Annual Review of Public Health, 1992; 13: 489-508. 
8. M. Tinetti, M. Speechley and S. Ginter: Risk factors for falls among elderly persons living in the community. New England Journal of Medicine, 1988; 319: 170-177.

9. M. Tinetti and M. Speechley: Prevention of falls among the elderly. New England Journal of Medicine, 1989; 320: 1055-1059.

10. B. J. McFadyen and F. Prince: Avoidance and accommodation of surface height changes by healthy, community-dwelling, young, and elderly men. Journals of Gerontology Series A Biological Sciences \& Medical Sciences, 2002; 57:

11. E. S. Grood and W. J. Suntay: A joint coordinate system for the clinical description of threedimensional motions: application to the knee. Journal of Biomechanical Engineering, 1983; 105: 136-144. 